\title{
Wavelet based Multimodal Biometrics with Score Level Fusion Using Mathematical Normalization
}

\author{
Priti S. Sanjekar \\ Department of Computer Engineering, R. C. Patel Institute of Technology, Shirpur and \\ KBC North Maharashtra University, Jalgaon, India \\ Email:priti_san2003@yahoo.com \\ J. B. Patil \\ Department of Computer Engineering, R. C. Patel Institute of Technology, Shirpur and \\ KBC North Maharashtra University, Jalgaon, India \\ Email: jbpatil@ hotmail.com
}

Received: 31 October 2018; Accepted: 18 December 2018; Published: 08 April 2019

\begin{abstract}
Biometric based authentication is playing a very important role in various security related applications. A novel multimodal biometric verification based on fingerprint, palmprint and iris with matching score level fusion using Mathematical Normalization is proposed in this paper. In feature extraction stage of unimodal, features of each modality are extracted by applying wavelet decomposition using 6 different wavelet families and 35 respective wavelet family members. Further, the three optimal combinations of unimodal systems based on equal error rate achieved by wavelet(s) are chosen for development of multimodal biometric system. In matching score level fusion, along with wellknown normalization techniques- Min-max, Tan-h and Zscore, the performance of multimodal systems are also analyzed using Mathematical Normalization (Math-norm) followed by product, weighted product, sum and average fusion rule. The experiments are conducted on database of 100 different subjects from publically available FVC2006, CASIA V1 and IITD database of fingerprint, palmprint and iris, respectively. The experimental results clearly show that Mathematical Normalization followed by weighted product has given promising accuracy with equal error rate (EER) of $0.325 \%$.
\end{abstract}

Index Terms-Multimodal biometrics, Matching score level fusion, mathematical normalization, wavelet, fingerprint, palmprint, iris.

\section{INTRODUCTION}

Biometrics based personal authentication is playing a significant role in various operations of security related applications. Biometrics provides potential benefits over possession and knowledge based methods [1]. Biometric system is a pattern recognition system which authenticates a person based on either his/her physiological attributes like fingerprint, palmprint, face, iris, ear, finger-vein, finger-knuckle, hand geometry, retina, DNA etc. or behavioral attributes like voice, signature, keystroke, gait etc. Biometric attribute should satisfy criteria like universality, time invariance, distinguishable, collectability, performance and acceptability [1]. Unimodal biometric authentication employs single attribute for verification or identification of a person. It faces some drawbacks like noisy data, spoofing risk, non universality, inter-class similarity and intra-class variation. To alleviate these problems, biometric research community is now gravitated towards multimodal biometric system [2].

Multimodal biometrics is attracting more attention as compared to the unimodal biometrics. The multimodal biometrics is more reliable and provides strong security. It verifies or identifies a person by combining evidences from two or more modals or attributes of person e.g. fingerprint-palmprint, fingerprint-fingervein-iris etc. These different biometric evidences acquired from various sources can be merged at feature level, score level or decision level. At feature level, the features of different biometric traits are integrated., at score level, the matching scores of different traits from different matchers are combined while in decision level, output of different traits (final identity or result of verification) are considered for final output $[3,4,5]$. The proper integration of such information from different modalities can be very helpful than using information from just single modality. The fusion at feature level, where the features combined from different modals may form high dimensional and redundant features also features from different traits may not be compatible. The fusion at decision level is computationally simple and easy. However, sometimes it cannot meet real time limitations due to lack of information [1]. The fusion at matching score level is shown to be most effective approach in multimodal biometrics. Proper fusion of the different characteristics is the key to success of multimodal biometrics [6].

In present work, multimodal biometric verification based on fingerprint, palmprint and iris with match score level fusion using mathematical normalization is proposed. These biometric characteristics are chosen 
because fingerprint is most widely used and accepted, palmprint has larger surface area with stable and discriminating features and iris has stable and unique structure which does not change over the lifetime [7]. In this work, the combination approach for fusion of the scores from different modalities is considered. This work will first examine the performance of multimodal biometric system over the unimodal biometric system. Further, the performance evaluation of multimodal system is based on well-known normalization techniques i.e. Min-Max, Tanh and Z-score normalizations with product, weighted product, sum and average fusion rules. Additionally, the main objective of the work is to evaluate performance of the system with mathematical normalization with various fusion methods. Many researchers have used normalization techniques like MinMax, Tanh, Z-score, Median-MAD, Double sigmoid etc. These techniques are used frequently in literatures but the mathematical normalization on the combination of fingerprint, palmprint and iris is not addressed yet. Therefore, the proposed work will focus and show the effectiveness of mathematical normalization.

The paper is structured as follows: Section 2 gives review of literatures; Section 3 describes fingerprint, palmprint and iris verification system based on 6 different wavelet families and 35 different wavelet members and matching score level fusion. Databases and experimental set up is covered in Section 4. Section 5 gives the performance of different normalization methods with various fusion techniques in terms of Equal Error Rate and Receiver Operating Characteristic. Finally, the conclusions are reported in last Section.

\section{LITERATURE REVIEW}

Till date many multimodal biometric systems based on modals like face-iris, fingerprint-finger vein-hand geometry, hand geometry-hand shape, palmprint-iris, fingervein-fingerknuckle etc. have been developed by various researchers but fingerprint, palmprint and iris based multimodal biometric system with matching score level fusion using mathematical normalization is yet not reported. Right choices of traits, feature extraction technique, fusion schemes, matching algorithm etc. are some open challenges in multimodal biometrics [8]. In this section survey of literature is explored.

In 2015, S. Sharma et al. [9] worked on the multibiometric system based on combination of shape and geometry of the hand to perform verification. The wavelet decomposition at level 5 with Daubechies wavelet 1 is carried out to reduce the dimensions of the distance and orientation features. They adopted a two level score level fusion in order to increase the performance of the verification and they also performed experiments with different similarity measures. They reported $0.52 \%$ EER on IITD database. S. Ribaric et al. [10] have developed multimodal biometric verification system using face and palm in 2006. They have developed verification and two identification systems based on face, palm and finger. Normalization of the scores is carried out with normalization techniques like piecewise linear, min-max, median-MAD, double sigmoid, tanh, zscore and bayes. For verification system with Bayes normalization authors have achieved minimum EER of $2.29 \%$, while they reported $3.12 \%, 3.05 \%$ and $3.15 \%$ EER for min-max, tanh and $\mathrm{z}$-score techniques respectively.

In 2016, T. A. Alghamdi et al. [11] presented a multimodal biometric system using face and palmprint. They evaluated this combination with different fusion schemes and experimental results show that fusion at score level is more effective. He has reported accuracy in GAR of $91 \%$ at 0.01 FAR with sum rule of matching score level fusion. In 2010, M. I. Razzak et al. [12] have proposed face and finger vein based multimodal biometric recognition system with classification based approach using fuzzy fusion on the database of 35 subjects. The considerable performance of $91.4 \%$ GAR at $0.05 \%$ FAR of their system is reported. In 2010, A. P. Yazdanpana et al. [13] have developed the face, ear and gait based multimodal biometric system and evaluated the performance of different normalization techniques like Min-Max, Median-MAD and Z-score with weighted sum and weighted product. The experimental results are obtained on the database of 40 subjects with system accuracy of $97.5 \%$ GAR at $0.1 \%$ FAR using Z-score with weighted product combination. In 2013, A. B. Khalifa et al. [14] proposed the novel approach of adaptive normalization for multimodal biometrics using face, fingerprint and palmprint. The experimentation is performed using various normalization schemes with different fusion methods on database of 100 users. The promising result with $0.47 \%$ EER is obtained for adaptive normalization and product rule.

In 2006, A. Kumar et al. [15] have proposed fingerprint, palmprint and hand shape based biometric system. These characteristics are from the same modalities i.e. hand alone and extracted features from fingerprint, palmprint and hand shape, then scores of individual features are simply combined using sum rule. The performance of the system is analyzed on the database of the 100 subjects collected by their own using digital camera and they got considerable performance with EER of 3.54\%. In 2011, M. Hanmandlu et al. [16] have introduced the approach of triangular norm for matching score level fusion on multimodal system of palmprint, hand veins and hand geometry which are the features of single modality i.e. hand alone. Here, the fusion rules (product and sum) rules are extended to general class of aggregation operators (t-norms) in order to enhance the score level fusion. The experimentation is conducted on two databases i.e. IITD and polyU of 100 and 165 subjects, respectively using triangular norm with result of $100 \%$ GAR at 0.01 FAR.

In 2010, He et al. [6] have evaluated the performance of the transformation based as well as classifier based score level fusion and analyzed the performance of wellknown normalization techniques i.e. Min-Max, Tanh and Zscore with sum rule. Further, they derived a new normalization technique-Reduction of High score Effect 
(RHE). They performed experiment on 510 subjects from NIST face and fingerprint database for fingervein virtual database is created. The system has accuracy of $99.8 \%$ at $0.01 \%$ FAR using RHE. In 2006, K. Nandakumar et al. [17] have proposed density based likelihood ratio score level fusion with taking quality of the image into account using fingerprint and iris of 310 users. The complex process for quality assessment is used with achieved GAR of $94.8 \%$ at $0.01 \%$ FAR. In the same year A. Jain et al. [18] have investigated score normalization in multimodal biometrics based on face, fingerprint and hand geometry. The performance of different normalization techniques with various fusion methods are experimented and analyzed on the database of 100 subjects. According to experiments min-max and z-score are sensitive to the outliers. At $0.1 \%$ FAR, for min-max, $\mathrm{z}$ -score and tanh author has achieved accuracy of $97.8 \%$, 98.6\% and $98.5 \%$ respectively.

In 2011, R. Raghavendra et al. [19] designed multimodal biometric system using face and palmprint along with score level fusion. The experimentations are also conducted on feature level fusion using particle swarm optimization. The matching score fusion is carried out with weighted sum rule. The experiments are carried out on 250 users from FRGS face database and PolyU palmprint database and they got accuracy of $86.50 \%$ at $0.01 \%$ FAR. In 2011, Cui et al. [20] have proposed score level fusion of fingerprint and fingervein. The minutia based matching is performed for fingerprint while modified hausdorff distance (MHD) algorithm is used for fingervein matching. With min-max normalization and weighted sum rule, weights are varied in range of $[0,1]$. They experimented on database of 80 subjects and got accuracy of $98.74 \%$ with 0.7 and 0.3 weights for finger and vein respectively. In 2014, M. Shahrimie et al. [21] proposed multimodal biometrics based on finger vein and finger geometry with BLPOC (Band Limited Phase Only Correlation) and WCCD (Width with Centroid Contour Distance) for matching. The $\mathrm{z}$-score normalization with weighted sum rule is employed for fusion of scores. Own collected database of 123 subjects are used for the experimentation to achieve the considerable results of $1.78 \%$ EER.

It is revealed from literature survey that mathematical normalization technique on fingerprint, palmprint and iris modals for score level fusion in multimodal system is yet not addressed. Therefore, this paper mainly focuses on mathematical normalization method in multimodal score level fusion with various combination of wavelet families in unimodal systems and also shows performance of the system with various values of alpha in mathematical normalization.

\section{PROPOSED METHOD}

\section{A. Fingerprint Feature Extraction}

In present work, the image based approach of fingerprint verification system is used. In image based approach generally features are extracted directly from raw gray scale image with no or minimum preprocessing steps. Gray-scale fingerprint image is much richer with discriminatory information. The minimum pre-processing requirements with this approach also reduces the computational complexity [22]. In order to extract the features from fingerprint image, first of all area of $250 \mathrm{X}$ 300 around the core point is considered [23], then Gray Level Co-occurrence Matrix (GLCM) and wavelet based features are extracted from the cropped area. The GLCM is computed from input fingerprint image using (1) for feature extraction. GLCM has proved to be popular statistical method of extracting texture features from images [24]. In this work four gray level co-occurrence matrices are calculated with four different angles i.e. $0^{0}$ (horizontal), $90^{\circ}$ (vertical), $45^{\circ}$ and $135^{\circ}$ (diagonal) and then statistical texture features namely, Contrast, Homogeneity, Energy and Correlation are extracted from these gray level co-occurrence matrices by using (2) to (5) respectively. For a given image I of size $\mathrm{N} \times \mathrm{N}, \mathrm{GLCM}$ is defined as

$$
P(i, j)=\sum_{x, y=1}^{N}\left\{\begin{array}{rr}
1, & \text { ifI }(x, y)=i \text { and } I\left(x+d_{x}, y+d_{y}\right)=j \\
0 & \text { otherwise }
\end{array}\right.
$$

Here, the offset $\left(d_{x}, d_{y}\right)$, specifies the distance between the pixel-of-interest and its neighbor.

$$
\begin{gathered}
\text { Contrast }=\sum_{\mathrm{i}, \mathrm{j}=1}^{\mathrm{k}}(\mathrm{i}-\mathrm{j})^{2} \mathrm{P}(\mathrm{i}, \mathrm{j}) \\
\text { Homogeneity }=\sum_{i, j=1}^{k} \frac{P(i, j)}{1+|i-j|} \\
\text { Energy }=\sum_{\mathrm{i}, \mathrm{j}=1}^{\mathrm{k}} \mathrm{P}(\mathrm{i}, \mathrm{j})^{2} \\
\text { Correlation }=\sum_{\mathrm{i}, \mathrm{j}=1}^{\mathrm{k}} \frac{(\mathrm{i}-\mu \mathrm{i})(\mathrm{j}-\mu \mathrm{j}) \mathrm{P}(\mathrm{i}, \mathrm{j})}{\sigma_{\mathrm{i}} \sigma_{\mathrm{j}}}
\end{gathered}
$$

Where $P$ is co-occurrence matrix, $P(i, j)$ is $(i, j)^{\text {th }}$ entry in co-occurrence matrix, $\mathrm{k}$ denotes dimension i.e. no. of gray levels of co-occurrence matrix, $\mu$ is mean and $\sigma$ is the standard deviation. The four features namely contrast, homogeneity, energy and correlation for each angle $\left(0^{0}\right.$, $45^{\circ}, 90^{\circ}$ and $135^{\circ}$ ) are extracted from four different GLCMs. Thus, at this point total 16 features are extracted from GLCMs.

The wavelet based features are also extracted from fingerprint along with texture features. The wavelet transformation is based on Multi-resolution analysis and represents signal in frequency and space [25]. The various wavelet components are the shifted and the scaled versions of a mother wavelet [26]. Here, the original fingerprint image is decomposed up to four levels. At each level of wavelet transformation only LH, HL and $\mathrm{HH}$ parts are considered for feature extraction and GLCM of this each part is computed with angle $0^{\circ}, 90^{\circ}, 45^{\circ}$ and $135^{\circ}$ respectively. For $\mathrm{HH}$ part, GLCMs are computed with two angles i.e. $45^{\circ}$ and $135^{\circ}$. Finally, from these GLCMs statistical texture features contrast and energy (32 features) are extracted using (2) and (4), respectively. 
The mean and standard deviation of detail parts of first level decomposition (6 features) are also extracted using wavelet transformation. In order to improve the accuracy, the wavelet energy of the detail components up to $5^{\text {th }}$ level decomposition (45 features) is also computed. In this way total 99 features are extracted from each fingerprint image. Note that for decomposition 6 different wavelet families namely Haar, Daubechies, Symlet, Coiflets, Bior and Dmeyer with their respective 35 wavelet members are used. For each wavelet member, accuracy of the fingerprint system in terms equal error rate is calculated which is shown in Table 1-6.

\section{B. Palmprint Feature Extraction}

The palmprint Region of Interest extraction is very crucial but important task in palmprint based authentication. It is extracted using center and centroid of the palmprint [27]. In proposed work, palmprint feature extraction is also based on wavelet transformation. Palmprint ROI of size $200 \times 200$ is decomposed at single level. In order to extract the local statistical features, block processing is performed on decomposed palm print image. Decomposed image is divided into blocks of size 10 by 10 resulted in 400 blocks. Then mean of each block is calculated using (6).

$$
\operatorname{mean}(\mathrm{m})=1 / \mathrm{N}^{2} \sum_{\mathrm{i}, \mathrm{j}=1}^{\mathrm{N}} \mathrm{I}(\mathrm{i}, \mathrm{j})
$$

Where $\mathrm{m}$ is the mean of block, $\mathrm{N}$ is dimension of block and $I(i, j)$ is $(i, j)^{\text {th }}$ pixel entry in that block. In this way the total 400 statistical features are extracted from decomposed palmprint image. Note that for palmprint decomposition also 6 different wavelet families namely Haar, Daubechies, Symlet, Coiflets, Bior and Dmeyer with their respective 35 wavelet members are used. For each wavelet member accuracy of the palmprint system is calculated as shown in Table 1-6. For both fingerprint and palmprint matching Euclidean distance is used which is calculated with (7)

$$
E D=\sqrt{\left(\sum_{i=1}^{n o . o f ~ f e a t u r e s}\left(f e a_{k, i}-f e a_{l, i}\right)^{2}\right)}
$$

Where ED is Euclidean distance and fea $a_{k, i}$ the features of unknown image, fea $\mathrm{a}_{1, \mathrm{i}}$ are features of known image from training database.

\section{Iris Feature Extraction}

The IITD iris database consists of normalized iris images [28]. These normalized images are considered for feature extraction. Iris feature extraction is also based on wavelet transformation. Normalized iris image is decomposed up to fourth level using wavelet transformation. Wavelet coefficients of fourth level decomposed LL, LH, HL and HH parts are selected as a feature vector, value of the coefficient can be positive or negative. The wavelet coefficients are encoded into 324 bit binary code using (8) where $\mathrm{V}$ is the feature vector and $\mathrm{V}(\mathrm{i})$ is $i^{\text {th }}$ bit of feature vector. For iris matching Hamming Distance [29] is used which is calculated with
(9)

$$
\begin{aligned}
V(i) & =1, \quad \text { if } V(i)>0 \\
V(i) & =0, \quad \text { otherwise } \\
H D(p, q) & =\frac{1}{N} \sum_{j=1}^{N}\left(p_{j \oplus q_{j}}\right)
\end{aligned}
$$

where $p$ and $q$ are feature codes of two iris, $p_{j}$ and $q_{j}$ is corresponding bit of two codes and $\mathrm{N}$ is size of features vector.

\section{Matching Score Level Fusion}

Matching score level fusion is a scheme of post classification information fusion [18]. The information fusion at matching score level is feasible [30]. Fusion at matching score level can be divided into three types: Transformation based fusion, Classifier based fusion and Density based fusion. In transformation based fusion, the matching scores from different modalities are transformed/normalized into same domain and then combined using various fusion rules like product, sum etc. In classifier based fusion, the scores from various modalities are combined and given as an input to classifier and lastly Density based fusion which is based on estimation of matching scores density either by parametric or non-parametric methods [5,19,31]). In present work, transformation based fusion is employed. As scores from different modalities are from different domain/ range, they need to be normalized in order to transform them into common domain. For normalization of the fingerprint, palmprint and iris scores, the three well known normalization techniques are employed as: MinMax, Tanh and Z-score normalization [18]. These normalization techniques are often appeared in the literatures. Besides this, the main contribution of this work is use of Mathematical normalization. Min-max, Tanh, Z-score and Mathematical normalization are performed using (10) to (13) respectively.

$$
x^{\prime}=\frac{x-\min (x)}{\max (x)-\min (x)}
$$

In Eq. (10), $\max (\mathrm{X})$ and $\min (\mathrm{X})$ are the maximum and minimum value from the raw scores. In Eq. (11), $\mu$ is the mean of genuine scores, $\sigma$ is the standard deviation of the genuine scores. Z-score normalization is given by (12) where $\mu$ is the mean of raw scores, $\sigma$ is the standard deviation of the raw scores, here $\mathrm{x}$ is raw score and $\mathrm{x}$ ' is the normalized score.

$$
\begin{gathered}
x^{\prime}=\frac{1}{2}\left\{\tanh \left(0.01\left(\frac{x-\mu}{\sigma}\right)\right)+1\right\} \\
x^{\prime}=\frac{(x-\mu)}{\sigma} \\
x^{\prime}=\frac{x}{\sqrt{x^{2}+\alpha}}
\end{gathered}
$$

Mathematical normalization is given by (13) [32], where $\mathrm{x}$ is raw score, $\alpha$ is the constant value like 50,100 
and $x^{\prime}$ is the normalized score. Next section covers databases used in the experiments.

\section{DATABASES AND EXPERIMENTAL SETUP}

In present work, the multimodal database used for experiments is heterogeneous or virtual database which is created by pairing a user from one unimodal database with user from another unimodal database. FVC2006 fingerprint database collected by Biometric System Lab (BIOLAB), University of Bologna with different sensors is used for fingerprint. All images are in .bmp format [33]. FVC2006 consist of 4 sets i.e. DB1, DB2, DB3 and DB4. For present study DB2_A set is used and it consists of fingers of 140 subjects. Out of 140 subjects, 100 subjects are randomly selected for experiments with 5 images per subject.

Publically available CASIA Palmprint V1 Database collected by the Chinese Academy of Sciences, Institute of Automation (CASIA) is used for palmprint [34]. CASIA palmprint V1 database contains the images of 312 subjects. These palmprint images are captured using CMOS camera. The palmprint experimentations are carried out on images of 100 subjects with 5 images per subjects. Finally, IITD Iris Image Database Version 1.0 acquired in the Biometrics Research Laboratory using JPC1000, JIRIS and digital CMOS camera is used for last modality i.e. iris [28]. This database consists of iris images of 224 subjects. For this work, 100 different iris images are randomly selected with 5 images per subjects. In all three modalities, out of 5 images of each subject, 3 images are used for training while remaining images are used for testing.

For multimodal biometrics experiments, these three unimodal databases are used and formed heterogeneous database so experiments are performed on total 1500 different images. From each three unimodal characteristics i.e. fingerprint, palmprint and iris, genuine and imposter scores were obtained. Each image of the subject is matched with remaining images of the same subject to obtain genuine scores while each image of the subject is matched with each image of the every other subject to produce imposter scores. In unimodal biometrics, 6 different wavelet families and 35 respective wavelet members are used for features extraction and accuracy of the system is measured in terms of False Acceptance Rate (FAR), False Rejection Rate (FRR) and Equal Error Rate (EER). EER is the point where FAR is equals to FRR. Generally, EER should be minimum for secure system. Next section covers the experimental results of both unimodal \& multimodal biometric system.

\section{EXPERIMENTAL RESULTS AND DISCUSSIONS}

The proposed work is implemented in MATLAB. The experimentation is carried out with three unimodal verification systems namely, fingerprint, palmprint and iris. Six different wavelet families namely, Haar, Daubechies, Symlet, Coiflets, Bior and Dmeyer along with their respective thirty five family members i.e. haar, $\mathrm{db} 2, \mathrm{db} 3, \mathrm{db} 4, \mathrm{db} 5, \mathrm{db} 6, \mathrm{db} 7, \mathrm{db} 8, \mathrm{db} 9, \mathrm{db} 10$, sym2, sym3, sym4, sym5, sym6, sym7, sym8, coif1, coif2, coif3, coif4, coif5, bior1.1, bior1.3, bior1.5, bior2.2, bior2.4, bior2.6, bior2.8, bior3.1, bior3.3, bior3.5, bior3.7, bior3.9 and dmey are used in feature extraction stage of each modality. The results of experimentation with Haar, Daubechies, Symlet, Coiflets, Bior and Dmeyer wavelet families are shown in Table 1-6, respectively. The accuracy of each unimodal system is measured in terms of Equal Error Rate (\%EER) which is determined from False Acceptance Rate (FAR) and False Rejection Rate (FRR). The minimum value of EER represents the high accuracy of unimodal and multimodal systems. The fingerprint unimodal system has achieved minimum EER of $5.6 \%$ using sym5 wavelet member among all wavelet members. Similarly, the palmprint unimodal system has achieved minimum EER of 5.5\% using db4, sym6, bior2. 4 and bior3.5 wavelet members among all wavelet members. Finally, the iris unimodal system has achieved minimum EER of $1.5 \%$ using haar and bior1.1wavelet members among all wavelet members.

Table 1.Unimodal Results with Haar Wavelet

\begin{tabular}{|c|c|c|c|c|}
\hline $\begin{array}{l}\text { Wavelet } \\
\text { Family }\end{array}$ & $\begin{array}{c}\text { Wavelet } \\
\text { family } \\
\text { member(s) }\end{array}$ & $\begin{array}{c}\text { Fingerprint } \\
\text { (\%EER) }\end{array}$ & $\begin{array}{c}\text { Palmprint } \\
\text { (\%EER) }\end{array}$ & $\begin{array}{c}\text { Iris } \\
(\% \text { EER) }\end{array}$ \\
\hline Haar & Haar & 6.4 & 5.8 & 1.5 \\
\hline
\end{tabular}

Table 2. Unimodal Results with Daubechies wavelet

\begin{tabular}{|c|c|c|c|c|}
\hline $\begin{array}{c}\text { Wavelet } \\
\text { Family }\end{array}$ & $\begin{array}{c}\text { Wavelet } \\
\text { family } \\
\text { member(s) }\end{array}$ & $\begin{array}{c}\text { Fingerprint } \\
\text { (\%EER) }\end{array}$ & $\begin{array}{c}\text { Palmprint } \\
\text { (\%EER) }\end{array}$ & $\begin{array}{c}\text { Iris } \\
\text { (\%EER) }\end{array}$ \\
\hline \multirow{5}{*}{$\begin{array}{c}\text { Daubechies } \\
\text { (db) }\end{array}$} & $\mathrm{db} 2$ & 6.3 & 5.9 & 2.5 \\
\cline { 2 - 5 } & $\mathrm{db} 3$ & 6.1 & 5.6 & 6.5 \\
\cline { 2 - 5 } & $\mathrm{db} 4$ & 6.1 & 5.5 & 7.4 \\
\cline { 2 - 5 } & $\mathrm{db} 5$ & 6.57 & 5.65 & 10.5 \\
\cline { 2 - 5 } & $\mathrm{db} 6$ & 6.2 & 5.65 & 7.7 \\
\cline { 2 - 5 } & $\mathrm{db} 7$ & 7.2 & 5.8 & 5.7 \\
\cline { 2 - 5 } & $\mathrm{db} 8$ & 8.1 & 6.2 & 9.7 \\
\cline { 2 - 5 } & $\mathrm{db} 9$ & 9.8 & 5.8 & 8 \\
\hline
\end{tabular}

Table 3. Unimodal Results with Symlet wavelet

\begin{tabular}{|c|c|c|c|c|}
\hline $\begin{array}{c}\text { Wavelet } \\
\text { Family }\end{array}$ & $\begin{array}{c}\text { Wavelet } \\
\text { family } \\
\text { member(s) }\end{array}$ & $\begin{array}{c}\text { Fingerprint } \\
\text { (\% EER) }\end{array}$ & $\begin{array}{c}\text { Palmprint } \\
\text { (\%EER) }\end{array}$ & $\begin{array}{c}\text { Iris } \\
\text { (\% EER) }\end{array}$ \\
\hline \multirow{4}{*}{$\begin{array}{c}\text { Symlets } \\
\text { (sym) }\end{array}$} & sym2 & 6.4 & 5.85 & 2.4 \\
\cline { 2 - 5 } & sym3 & 6.1 & 5.7 & 6.7 \\
\cline { 2 - 5 } & sym4 & 6.9 & 5.6 & 4.25 \\
\cline { 2 - 5 } & sym5 & 5.6 & 5.65 & 5.5 \\
\cline { 2 - 5 } & sym6 & 7.4 & 5.5 & 3.5 \\
\cline { 2 - 5 } & sym7 & 6.4 & 5.6 & 8 \\
\hline
\end{tabular}

Table 4. Unimodal Results with Coiflet wavelet

\begin{tabular}{|c|c|c|c|c|}
\hline $\begin{array}{c}\text { Wavelet } \\
\text { Family }\end{array}$ & $\begin{array}{c}\text { Wavelet } \\
\text { family } \\
\text { member(s) }\end{array}$ & $\begin{array}{c}\text { Fingerprint } \\
\text { (\% EER) }\end{array}$ & $\begin{array}{c}\text { Palmprint } \\
\text { (\%EER) }\end{array}$ & $\begin{array}{c}\text { Iris } \\
\text { (\% EER) }\end{array}$ \\
\hline \multirow{4}{*}{$\begin{array}{c}\text { Coiflet } \\
\text { (coif) }\end{array}$} & coif1 & 6.25 & 5.6 & 6.25 \\
\cline { 2 - 5 } & coif2 & 6.8 & 5.6 & 13.2 \\
\cline { 2 - 5 } & coif3 & 6.75 & 5.8 & 9.9 \\
\cline { 2 - 5 } & coif4 & 9 & 5.8 & 7.4 \\
\cline { 2 - 5 } & coif5 & 9.6 & 5.8 & 6.75 \\
\hline
\end{tabular}

I.J. Image, Graphics and Signal Processing, 2019, 4, 63-71 
The summation of equal error rates (\% EER) of the fingerprint, palmprint and iris is calculated for respective wavelet members of each wavelet family. Further, the top two combinations having minimum summation of equal error rate are selected for development of multimodal biometric system using matching score level fusion. In first combination of multimodal, the bior1.1 wavelet while in second combination, Haar wavelet has given lowest summation of \% EER. The combination of three unimodal systems with lowest equal error rate (\% EER) is also selected for development of multimodal biometric system. Thus, sym5 wavelet based fingerprint unimodal, $\mathrm{db} 4$ wavelet based palmprint unimodal and haar wavelet based iris unimodal are combined to form third combination for multimodal system. The selected three combinations of unimodal for development of three different multimodal biometric systems and their respective unimodal results are as shown in Table 7.

Table 5.Unimodal Results with Bior wavelet

\begin{tabular}{|c|c|c|c|c|}
\hline $\begin{array}{c}\text { Wavelet } \\
\text { Family }\end{array}$ & $\begin{array}{c}\text { Wavelet } \\
\text { family } \\
\text { member(s) }\end{array}$ & $\begin{array}{c}\text { Fingerprint } \\
\text { (\%EER) }\end{array}$ & $\begin{array}{c}\text { Palmprint } \\
\text { (\%EER) }\end{array}$ & $\begin{array}{c}\text { Iris } \\
(\text { \% EER) }\end{array}$ \\
\hline \multirow{5}{*}{$\begin{array}{c}\text { BiorSplines } \\
\text { (bior) }\end{array}$} & bior1.1 & 6.4 & 5.75 & 1.5 \\
\cline { 2 - 5 } & bior1.3 & 6.6 & 5.85 & 4 \\
\cline { 2 - 5 } & bior1.5 & 8.5 & 5.7 & 6.6 \\
\cline { 2 - 5 } & bior2.2 & 7.5 & 5.6 & 6 \\
\cline { 2 - 5 } & bior2.4 & 7.9 & 5.5 & 10.3 \\
\cline { 2 - 5 } & bior2.6 & 6.4 & 5.6 & 8.8 \\
\cline { 2 - 5 } & bior2.8 & 9 & 5.6 & 12.5 \\
\cline { 2 - 5 } & bior3.1 & 14.2 & 5.8 & 7.25 \\
\cline { 2 - 5 } & bior3.3 & 8.7 & 5.6 & 12 \\
\cline { 2 - 5 } & bior3.5 & 8.7 & 5.5 & 9.25 \\
\cline { 2 - 5 } & bior3.7 & 9 & 5.55 & 8.75 \\
\hline & bior3.9 & 14 & 5.6 & 79.5 \\
\hline
\end{tabular}

Table 6. Unimodal Result with DMeyer wavelet

\begin{tabular}{|c|c|c|c|c|}
\hline $\begin{array}{l}\text { Wavelet } \\
\text { Family }\end{array}$ & $\begin{array}{c}\text { Wavelet } \\
\text { family } \\
\text { member(s) }\end{array}$ & $\begin{array}{c}\text { Fingerprint } \\
\text { (\%EER) }\end{array}$ & $\begin{array}{c}\text { Palmprint } \\
\text { (\%EER) }\end{array}$ & $\begin{array}{c}\text { Iris } \\
(\% \text { EER) }\end{array}$ \\
\hline $\begin{array}{c}\text { DMeyer } \\
\text { (dmey) }\end{array}$ & Dmey & 10.7 & 7.3 & 3 \\
\hline
\end{tabular}

Table 7. Unimodal Cases Selected for Three Different Multimodal Biometric Systems

\begin{tabular}{|c|c|c|c|c|}
\hline $\begin{array}{c}\text { Multimodal } \\
\text { Biometric } \\
\text { System No. }\end{array}$ & Wavelet(s) & $\begin{array}{c}\text { Fingerprint } \\
\text { (\% EER) }\end{array}$ & $\begin{array}{c}\text { Palmprint } \\
\text { (\% EER) }\end{array}$ & $\begin{array}{c}\text { Iris } \\
\text { (\%EER) }\end{array}$ \\
\hline 1 & $\begin{array}{c}\text { bior1.1, } \\
\text { bior1.1, } \\
\text { bior1.1(F- } \\
\text { P-I) }\end{array}$ & 6.4 & 5.75 & 1.5 \\
\hline 2 & $\begin{array}{c}\text { haar, haar, } \\
\text { haar (F-P-I) }\end{array}$ & 6.4 & 5.8 & 1.5 \\
\hline 3 & $\begin{array}{c}\text { sym5, db4, } \\
\text { haar (F-P-I) }\end{array}$ & 5.6 & 5.5 & 1.5 \\
\hline
\end{tabular}

The three different multimodal biometric systems are developed for three combinations of unimodal systems using matching score level fusion. The matching score level fusion is carried out for above three combinations using normalization methods as Min-max, Z-score, Tanh and Mathematical normalization with fusion techniques as product, weighted product, sum and average. The results of multimodal biometric system No. 1 using bior1.1 wavelet for fingerprint, palmprint and iris are shown in Table 8 . The results of multimodal biometric system No. 2 using haar wavelet for fingerprint, palmprint and iris are shown in Table 9 while the results of multimodal biometric system using sym5, db4 and haar wavelet for fingerprint, palmprint and iris respectively are shown in Table 10.

The present work mainly focus on the performance evaluation of mathematical normalization technique. Various experiments are conducted for the evaluation of performance of Mathematical Normalization to improve the accuracy of multimodal systems by varying the value of mathematical constant (alpha) from 0 to 5000 in Eq. 13 It is represented graphically in Fig.1. It is observed from Fig. 1 that the value of mathematical constant (alpha) at 1000 gives the more accuracy and lowest \%EER. So the value of alpha as 1000 is selected for mathematical normalization. The normalization of the raw scores is carried out using (10), (11), (12) and (13) of Min-max, Tanh, Z-score and Mathematical normalization, respectively. In case of weighted product fusion method, the Equal Error Rates (\%EER) of unimodal systems are considered for weight calculation. The weight $\mathrm{W}_{\mathrm{p}}$ of fingerprint, palmprint and iris is calculated using (14). Here, $\mathrm{EER}_{\mathrm{q}}$ is the equal error rate of $\mathrm{q}^{\text {th }}$ modal and $\mathrm{n}$ represents number of modals used in fusion.

$$
W p=\frac{1 / E E R p}{\sum_{q=1}^{n}(1 / E E R q)}
$$

Table 8. Results of Multimodal Biometric System No.1 using Different Normalization Techniques

\begin{tabular}{|c|c|c|c|c|}
\hline \multirow{2}{*}{$\begin{array}{c}\text { Normalization } \\
\text { Techniques }\end{array}$} & \multicolumn{4}{|c|}{$\begin{array}{c}\text { Fusion methods } \\
\text { (\%EER) }\end{array}$} \\
\cline { 2 - 5 } & Product & $\begin{array}{c}\text { Weighted } \\
\text { product }\end{array}$ & Sum & Average \\
\hline Min-Max & 0.375 & 0.4 & 0.974 & 0.973 \\
\hline Tanh & 1 & 0.98 & 1 & 0.94 \\
\hline Zscore & 1 & 1 & 1 & 1 \\
\hline Math-norm & 0.45 & $\mathbf{0 . 3 2 5}$ & 1.5 & 1.5 \\
\hline
\end{tabular}

Table 9. Results of Multimodal Biometric System No.2 using Different Normalization Techniques

\begin{tabular}{|c|c|c|c|c|}
\hline \multirow{2}{*}{$\begin{array}{c}\text { Normalization } \\
\text { Techniques }\end{array}$} & \multicolumn{4}{|c|}{$\begin{array}{c}\text { Fusion methods } \\
\text { (\%EER) }\end{array}$} \\
\cline { 2 - 5 } & Product & $\begin{array}{c}\text { Weighted } \\
\text { product }\end{array}$ & Sum & Average \\
\hline Min-Max & 0.381 & 0.4 & 0.975 & 0.973 \\
\hline Tanh & 1 & 0.98 & 1 & 0.95 \\
\hline Zscore & 1 & 1 & 1 & 1 \\
\hline Math-norm & 0.46 & 0.331 & 1.5 & 1.5 \\
\hline
\end{tabular}

Table 10. Results of Multimodal Biometric System No.3 using Different Normalization Techniques

\begin{tabular}{|c|c|c|c|c|}
\hline \multirow{2}{*}{$\begin{array}{c}\text { Normalization } \\
\text { Techniques }\end{array}$} & \multicolumn{4}{|c|}{$\begin{array}{c}\text { Fusion methods } \\
\text { (\%EER) }\end{array}$} \\
\cline { 2 - 5 } & Product & $\begin{array}{c}\text { Weighted } \\
\text { product }\end{array}$ & Sum & Average \\
\hline Min-Max & 0.465 & 0.456 & 1 & 1 \\
\hline Tanh & 1 & 1 & 1 & 0.97 \\
\hline Zscore & 1.2 & 1.2 & 1 & 1 \\
\hline Math-norm & 0.62 & 0.49 & 1.5 & 1.5 \\
\hline
\end{tabular}


From Table 8, Table 9 and Table 10, it is clearly observed that lowest equal error rate (\%EER) $0.325 \%$, $0.331 \%$ and $0.456 \%$ are obtained for multimodal biometric system No. 1, 2 and 3 respectively. Further, among all these, the lowest EER of $0.325 \%$ is achieved using mathematical normalization technique with weighted product fusion method of Multimodal biometric system No. 1. The performance of the multimodal biometric system No.1 using mathematical normalization followed by weighted product fusion method in terms of equal error rate (ERR) is represented in Fig.2. ROC is plotted by FAR vs. GAR where GAR is Genuine Acceptance Rate (GAR = 100 - FRR). Fig.3 shows unimodal as well as multimodal results and it clearly indicates that multimodal system outperforms unimodal systems. The system gives $98.7 \%$ genuine acceptance rate (GAR) at $0.01 \%$ a false acceptance rate (FAR).

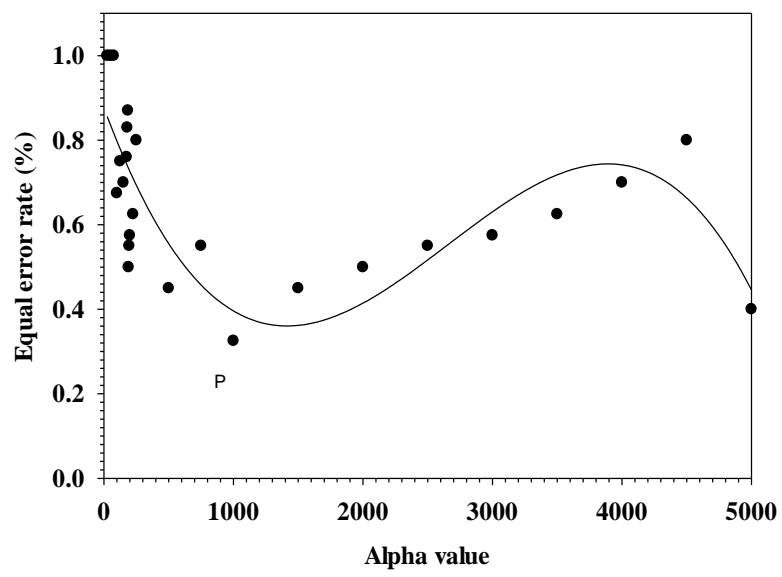

Fig.1. Performance of system with different values of alpha

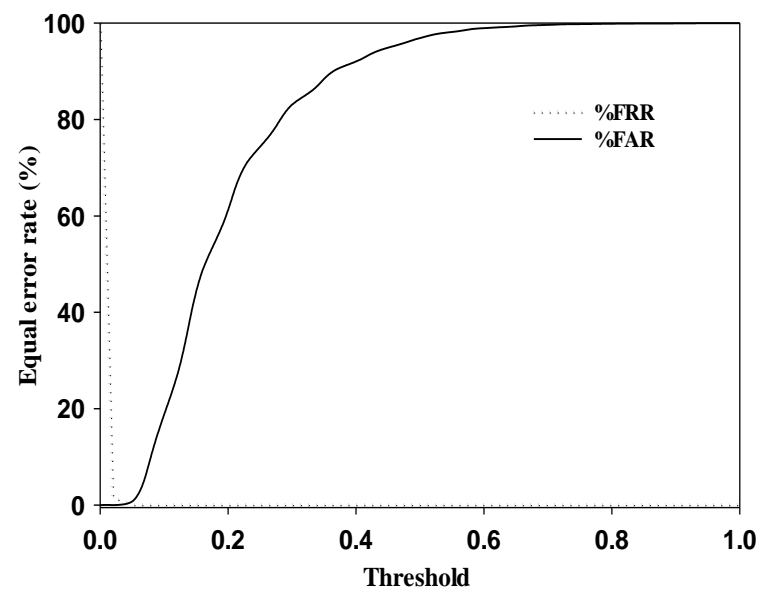

Fig.2. EER (\%) of multimodal biometrics using mathematical normalization with weighted product
The comparison of performance of proposed method with state-of-art methods in terms of the EER is reported in Table11. The Table 11 clearly shows that mathematical normalization gives best performance compared to wellknown methods like min-max, tanh and z-score.

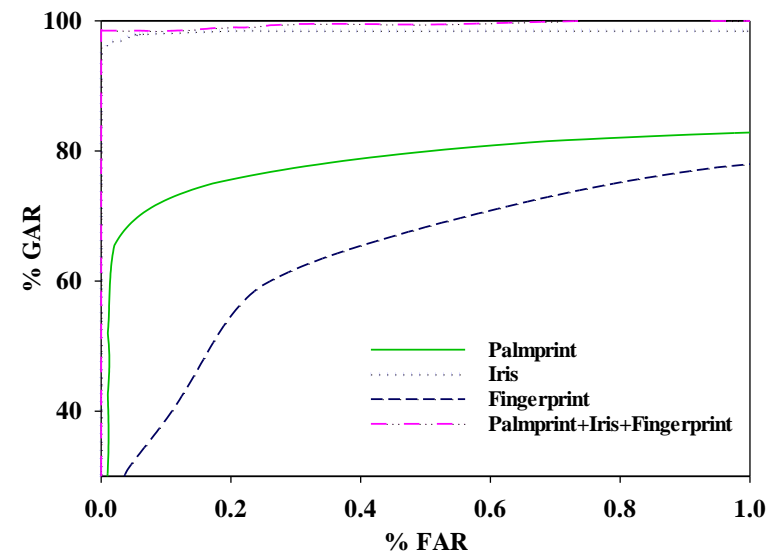

Fig.3. ROC curve of unimodal biometrics and multimodal biometrics

\section{CONCLUSIONS}

The multimodal biometric verification system based on fingerprint, palmprint and iris at matching score level fusion using mathematical normalization is presented. Development of the proposed multimodal biometric verification system is based on use of 6 different wavelet families and 35 respective wavelet family members in feature extraction stage of unimodal system. The performance of the system at matching score level fusion is analyzed using min-max, tan-h and $\mathrm{z}$-score normalization followed by product, weighted product, sum and average fusion methods. Along with these well known normalization methods, performance of the system is measured using mathematical normalization. Mathematical normalization is computationally very efficient and it is insensitive to outliers of the data. The best EER of $0.325 \%$ is achieved using mathematical normalization followed weighted product fusion for system which is based on use of bior1.1 wavelet for all fingerprint, palmprint and iris. Further it is also observed that mathematical constant-alpha in mathematical normalization gives best performance for large values. The proposed system gives $98.7 \%$ of GAR at a $0.01 \%$ FAR, so it can be used for personal authentication task. 
Table 11. Comparisons of State-of-art Methods in term of Equal Error Rate

\begin{tabular}{|c|c|c|c|c|}
\hline Reference & Modality & $\begin{array}{c}\text { No. of } \\
\text { subjects }\end{array}$ & Normalization & Performance $(\%)$ \\
\hline $\begin{array}{l}\text { M. S. M. Asaari et al. [21] } \\
\text { (2014) }\end{array}$ & $\begin{array}{c}\text { Finger vein } \\
\text { Finger geometry }\end{array}$ & 123 & Z-score + weighted sum & $\mathrm{EER}=1.78$ \\
\hline A. B. Khalifa et al.[14] (2013) & $\begin{array}{c}\text { Face } \\
\text { Palmprint } \\
\text { Fingerprint }\end{array}$ & 100 & $\begin{array}{c}\text { Adaptive }+ \text { mean } \\
\text { Adaptive }+ \text { min } \\
\text { Adaptive }+ \text { max } \\
\text { Adaptive }+ \text { product }\end{array}$ & $\begin{aligned} \mathrm{EER} & =0.48 \\
\mathrm{EER} & =2.49 \\
\mathrm{EER} & =2.46 \\
\mathrm{EER} & =0.47\end{aligned}$ \\
\hline $\begin{array}{l}\text { V. Kanhangad et al. [35] } \\
\text { (2011) }\end{array}$ & $\begin{array}{l}\text { 2D Hand Geometry } \\
\text { 3D Hand Geometry }\end{array}$ & 177 & Min-max + weighted sum & $\mathrm{EER}=2.3$ \\
\hline A. Kumar et al. [15] (2006) & $\begin{array}{c}\text { Fingerprint } \\
\text { Palmprint } \\
\text { Handshape }\end{array}$ & 100 & Sum & $\mathrm{EER}=3.53$ \\
\hline S. Sharma et al.[9] (2009) & $\begin{array}{c}\text { Hand shape } \\
\text { Hand Geometry }\end{array}$ & 100 & Min-max & $\mathrm{EER}=0.52$ \\
\hline S. Ribaric et al. [10] (2006) & $\begin{array}{l}\text { Palm } \\
\text { Face }\end{array}$ & 231 & $\begin{array}{c}\text { Min-max + sum } \\
\text { Tanh + sum } \\
\text { Z-score + sum }\end{array}$ & $\begin{array}{l}\mathrm{EER}=3.12 \\
\mathrm{EER}=3.05 \\
\mathrm{EER}=3.15\end{array}$ \\
\hline Proposed method & $\begin{array}{l}\text { Fingerprint } \\
\text { Palmprint } \\
\text { Iris }\end{array}$ & 100 & Math-norm+ weighted product & $\mathrm{EER}=0.325$ \\
\hline
\end{tabular}

\section{REFERENCES}

[1] J. A. Unar, W. C. Seng and A. Abbasi, "A Review of Biometric Technology Along with Trends and Prospects," Pattern Recognition, vol. 47, no. 8, pp. 2673-2688, August 2014.

[2] M. Hanmandlu, J. Grover, A. Gureja and H. M. Gupta, "Score level fusion of multimodal biometrics using triangular norms," Pattern Recognition Letters, vol. 32, no 14, pp.1843-1850, 2011.

[3] A. K. Jain, A. Ross and S. Prabhakar, "An Introduction to Biometric Recognition," IEEE Transactions on Circuits and Systems for Video Technology, vol. 14, no. 1, pp.4-19, 2004.

[4] A. K. Jain, P. Flynn and A. A. Ross, "Handbook of Multibiometric," Springer, N J, USA, pp. 1-22, 2008.

[5] J. Peng, A. Ahmed, and X. Niu, "Multimodal biometric authentication based on score level fusion of finger biometrics," Elsevier, Optik, vol. 125, pp. 6891-6897, 2014.

[6] M. He, S. Horng, P. Fan, R. Run, R. Chen, J. L. Lai, M. K. Khan and K. O. Sentosa, "Performance evaluation of score level fusion in multimodal biometric systems," Pattern Recognition, vol. 43, pp.1789-1800, 2010.

[7] A. Poursaberi and B. N. Arabi "A novel IRIS recognition system using morphological edge detector and wavelet phase features," Journal of Graphics, Vision and Image Processing, vol.6, pp. 9-15, 2005.

[8] P. S. Sanjekar and J. B. Patil, "An Overview of Multimodal Biometrics," Signal and Image Processing: An International Journal (SIPIJ), vol. 4, no.1, pp. 57-64, Feb 2013.

[9] S. Sharma, S. R. Dubey, S. K. Singh, R. Saxena and R. Singh, "Identity verification using shape and geometry of human hands," Expert Systems with Applications, vol. 42, pp. 821-832, 2015.

[10] S. Ribaric and I. Fratric, "Experimental Evaluation of Matching-Score Normalization Techniques on Different Multimodal Biometric Systems," Proc. IEEE Mediterranean Electrotechnical Conference, May 2006, pp. $498-501$.

[11] T. A. Alghamdi, "Evaluation of Multimodal Biometrics at Different Levels of Face and Palm Print Fusion
Schemes," Asian Journal of Applied Sciences, vol.9, no.2 pp.126-130, 2016.

[12] M. I. Razzak, R. Yusof and M. Khalid, "Multimodal face and finger veins biometric authentication," Scientific Research and Essays, vol. 5, no.17, pp. 2529-2534, Sep. 2010.

[13] A. P. Yazdanpanah , K. Faez and R. Amirfattahi, "Multimodal Biometric System using Face, Ear and Gait Biometrics," Proc.10th International IEEE Conference on Information Science, Signal Processing and their Applications (ISSPA), May 2010, pp.251-254.

[14] A. B. Khalifa, " Adaptive Score Normalization: A Novel approach for Multimodal Biometric Systems," World Academy of Science, Engineering and Technology, International Journal of Computer and Information Engineering, vol. 7, no. 3, pp.376-384, 2013.

[15] A. Kumar, D. Zhang, "Combining Fingerprint, Palmprint and Hand-Shape for User Authentication," Proc.18th International Conference on Pattern Recognition (ICPR), Aug. 2006, pp. 549-553.

[16] M. Hanmandlu, J. Grover, A. Gureja and H.M. Gupta, "Score level fusion of multimodal biometrics using triangular norms," Pattern Recognition Letters, vol.32, pp.1843-1850, 2011.

[17] K. Nandakumar, Y. Chen, A.K. Jain and S.C. Dass, "Quality based Score Level Fusion in Multibiometric systems," Proc. 18th International Conference on Pattern Recognition (ICPR), 2006, pp. 473-476.

[18] A. Jain, K. Nandakumar and A. Ross, "Score normalization in multimodal biometric systems," Pattern Recognition, vol. 38, pp. 2270 - 2285, 2005.

[19] R. Raghavendra, B. Dorizzi, A. Rao and G.H. Kumar, "Designing efficient fusion schemes for multimodal biometric systems using face and palmprint," Pattern Recognition, vol. 44, pp.1076-1088, 2011.

[20] F. F. Cui, and G. P. Yang, "Score Level Fusion of Fingerprint and Finger Vein Recognition," Journal of Computational Information Systems, vol. 16, pp. 57235731, 2011

[21] M. S. M. Asaari, S. A. Suandi and B. A. Rosdi, "Fusion of Band Limited Phase Only Correlation and Width Centroid Contour Distance for finger based biometrics," Expert Systems with Applications, vol. 41, pp. 3367-3382, 2014. 
[22] P. S. Sanjekar and P. S. Dhabe, "Fingerprint Verification Using Haar Wavelet", Proc. IEEE second International Conference on Computer Engineering and Technology (ICCET), vol. 3 no.1, 2010, pp.361-365.

[23] P. S. Sanjekar, P. D. Saraf and B. D. Patil, "Review on Core Point Detection Techniques in Fingerprint," International Journal Computer Applications, no.3, pp.18-20, Dec. 2014.

[24] P. Mohanaiah, P. Sathyanarayana and L. GuruKumar, "Image Texture Feature Extraction Using GLCM Approach", International Journal of Scientific and Research Publications. vol. 3 no. 5, pp. 1-5, May 2013.

[25] R. M. Rao and A. S. Bopardikar, "Wavelet Transforms: Introduction to theory and Applications," Addison Wesley Longman, Inc., Reading, 1998.

[26] G. Bhatnagar, Q. M. J. Wu and B. Raman, "A New Fractional Random Wavelet Transform for Fingerprint Security," IEEE Trans. On Systems, Man, and Cybernetics-Part A: Systems and Humans, vol. 42, no. 1, pp. 262-275, Jan. 2012.

[27] P. S. Sanjekar and J. B. Patil, "Method of ROI Extraction for Palmprint," Indian Patent Application No. 201621044219 A, Filed on $26^{\text {th }}$ Dec., 2016, Published on $24^{\text {th }}$ Feb., 2017.

[28] Iris http://web.iitd.ac.in/ biometrics/Database_Iris.html

[29] J. Daugman, "High confidence visual recognition of persons by a test of statistical independence," IEEE Trans. Pattern Analysis Machine Intelligence, vol. 15, pp. 11481161, Nov. 1993.

[30] S.C. Dass, K. Nandakumar and A.K. Jain, "A principled approach to score level fusion in multimodal biometric systems," Proc. $5^{\text {th }}$ International Conference on Audio and Video Based Personal Authentication (AVBPA), July 2005, pp. 1049-1058.

[31] R. O. Duda, P. E. Hart and D. G. Stork, "Pattern Classification," John Wiley \& Sons, New York, 2001.

[32] http://people.revoledu.com/kardi/tutorial/ Similarity/Normalization.html

[33] Fingerprint http://atvs.ii.uam.es/atvs/fvc2006.html
[34] Palmprint database: http://biometrics.idealtest.org

[35] V. Kanhangad, A. Kumar and D. Zhang, "A Unified Framework for Contactless Hand Verification," IEEE Trans. on information forensics and and Security, vol.6, no.3, Sep. 2011.

\section{Authors' Profiles}

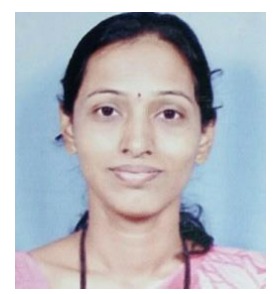

Priti S. Sanjekar has completed Master of Engineering in Computer Science and Engineering (IT) from VIT, Pune, Maharashtra, India. She is research scholar of KBC North Maharashtra University, Jalgaon and working as an Assistant professor at R. C. Patel Institute of Technology, Shirpur, Maharashtra, India. Her area of research is Biometrics and Image Processing.

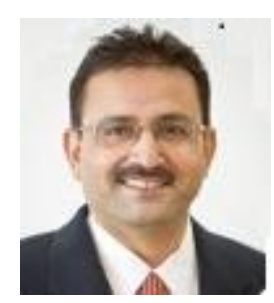

Jayantrao B. Patil has completed Master of Technology in Computer Science \& Data Processing from IIT, Kharagpur and Ph.D. in computer engineering from KBC North Maharashtra University, Jalgaon, Maharashtra, India. His area of research is web catching and web prefetching, web data mining, text watermarking, web usage mining, web personalization, semantic web mining, web security and image processing. He has published many research papers in International/National conferences and journals. He is a life member of Indian Society for Technical Education (ISTE), Computer Society of India (CSI), the member of Institute of Engineers (IE), India and the senior member of International Association of Computer Science and Information Technology (IACSIT), Singapore.

How to cite this paper: Priti S. Sanjekar, J. B. Patil, " Wavelet based Multimodal Biometrics with Score Level Fusion Using Mathematical Normalization", International Journal of Image, Graphics and Signal Processing(IJIGSP), Vol.11, No.4, pp. 63-71, 2019.DOI: 10.5815/ijigsp.2019.04.06 